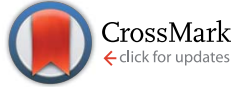

Cite this: RSC Adv., 2015, 5, 83187

Received 14th July 2015

Accepted 24th September 2015

DOI: $10.1039 / c 5 r a 13822 a$

www.rsc.org/advances

\section{Modulation of surface bio-functionality by using gold nanostructures on protein repellent surfaces $\uparrow$}

Sinan K. Muldur, ${ }^{\text {ab }}$ Cloe Desmet, ${ }^{a}$ Rita La Spina, ${ }^{a}$ Beatriz de Jesus da Cruz Monteiro, ${ }^{a}$ Blanka Halamoda-Kenzaoui, ${ }^{a}$ Valentina Spampinato, ${ }^{a}$ Giacomo Ceccone, ${ }^{a}$ Andrea Valsesia, ${ }^{a}$ Agnieszka Kinsner-Ovaskainen, ${ }^{a}$ Pascal Colpo ${ }^{\star a}$ and François Rossi ${ }^{a}$

The integration of gold nanoparticles (Au NPs) or nanostructures on solid surfaces for developing nanostructured biointerfaces has become a major research topic in the field of nanobiotechnology in particular for the development of a new generation of multifunctional bioanalytical platforms. This has led to considerable research efforts for developing quick and direct nanofabrication methods capable of producing well-ordered 2D nanostructured arrays with tunable morphological, chemical and optical properties. In this paper, we propose a simple and fast nanofabrication method enabling the creation of Au NP patterns on a non-adhesive and cell repellent plasma-deposited poly(ethyleneoxide) (PEO-like) coating. The immobilization of Au NPs on PEO-like coatings does not require any prior chemical modifications. By varying the size and the concentration of the Au NPs it is possible to control the Au NP number and density, and the average inter-particle distance on the PEO-like coated surface with direct effects on the bio-functionality of the surface. These nanostructured surfaces have been tested for protein bio-recognition analysis and as a cell culture platform. The developed nanostructured platform has many potential applications in the field of protein-nanoparticle and cell-nanoparticle interaction studies, nanotoxicology and bioengineering.

\section{Introduction}

Gold nanostructures can be used advantageously in many fields of applications such as biosensing, medical diagnostics and cell biology. ${ }^{1,2}$ Indeed, the integration of gold nanoparticles (Au NPs) or nanostructures on solid surfaces for the development of the next generation bio interfaces and analysis platforms has become a major research topic in the field of nanobiotechnology. Main efforts were devoted to the development and optimization of different nanofabrication techniques with the goal of obtaining a rapid and low cost method of fabrication with versatility features in terms of material types and geometric properties.

Among the different nanofabrication techniques, selfassembly is generally recognized as one of the most practical

${ }^{a}$ European Commission Joint, Research Centre, Institute for Health and Consumer Protection, Nanobioscience Unit, Via Fermi 1, 21027, Ispra, VA, Italy. E-mail: pascal.colpo@ec.europa.eu; Tel: +390332789979

${ }^{b}$ Institut de Chimie et Biochimie Moléculaires et Supramoléculaires, Equipe GEMBAS, Université Lyon 1, Bâtiment CPE 43, bd du 11 Novembre 1918, 69622 Villeurbanne Cedex, France

$\dagger$ Electronic supplementary information (ESI) available: Surface characterization of PEO-like coatings by X-ray Photoelectron Spectroscopy (XPS), ellipsometry, Contact Angle (CA). Au NPs characterization by Centrifugal Liquid Sedimentation (CLS), by dynamic light scattering and by Scanning Electron Microscopy (SEM). See DOI: 10.1039/c5ra13822a and easy methods for obtaining large area nanostructured surfaces with sub-micrometer resolution. ${ }^{3}$ More importantly, self-assembly techniques enable the straightforward creation of surface chemical nanopatterns, where at least two materials are patterned on the surface at the nano-scale to form nanocomposites. Nanocomposites can display a variety of features, such as electronic, optical and catalytic properties, depending on the nature, size, shape and distribution of the nanostructures. Self-assembly of nanoparticles of different sizes and densities has been widely used for designing detection platforms to enhance the sensitivity and/or the specificity of the detection. Several groups have highlighted the benefit of controlling the spatial organization of immobilized gold nanoparticles (Au NPs) to tune and improve considerably their localized Surface Plasmon Resonance (SPR).,5 Gold nanoparticles with controlled spatial distribution on surfaces have shown high potential for studying the mechanisms of cell adhesion and cell spreading on surfaces. ${ }^{6-9}$ Consequently, the possibility of specifically patterning metallic NPs onto a substrate to form ordered arrays gives new opportunities for developing highly functional and efficient analysis devices.

In this paper, we present a simple and direct method for creating large-area gold nanostructured patterns on a cell and protein repellent platform. The non-adhesive platform consists of plasma-deposited poly(ethyleneoxide) (PEO-like) coating which has already demonstrated high stability in biological 
environment such as cell culture conditions. ${ }^{\mathbf{1 0 - 1 2}}$ The Au NPs nanostructures are made by two methods, namely by direct spotting or by flowing NPs suspension on PEO-like coated surfaces. Surface distribution and coverage of Au NPs on PEOlike surface as a function of the NPs size and concentration were investigated by Scanning Electron Microscopy (SEM) and monitored in real-time using a Quartz Crystal Microbalance with Dissipation Monitoring technique (QCM-D). The suitability of this nanostructured platform for molecular detection was verified by monitoring protein interactions (IgG/anti-IgG) by QCM-D technique. Furthermore, we demonstrated the possibility of creating distinct patterns of Au NPs with a controlled number of anchoring points for cell adhesion and growth on PEO-like coatings, enabling to control the number of cells in each of the patterned micro-domains.

\section{Results and discussion}

\section{Immobilization of Au NPs on PEO-like coatings}

Deposition of $\mathrm{Au}$ NPs has been performed by using NPs suspension with decreasing concentrations of gold (0.5-0.250.125 and $0.063 \mathrm{mM}$ ) in citrate buffer ( $\mathrm{pH}$ 6.5). The suspensions of $15 \mathrm{~nm}$ size Au NPs were prepared and spotted (with a drop of $0.5 \mu$ l forming a spot of $1 \mathrm{~mm}^{2}$ in diameter) onto a PEO-like coated sample. The evaporation rate of each drop was controlled in order to ensure the same time of adsorption of $\mathrm{Au}$ NPs to the surface and to limit the "coffee ring" effect. SEM images of $15 \mathrm{~nm} \mathrm{Au} \mathrm{NPs} \mathrm{spots} \mathrm{show} \mathrm{a} \mathrm{well-dispersed} \mathrm{organi-}$ zation of NPs for each concentration tested (Fig. 1A-F).

As expected, decreasing the concentration i.e. the number of $\mathrm{Au}$ NPs, results in a decrease of their surface coverage per spot
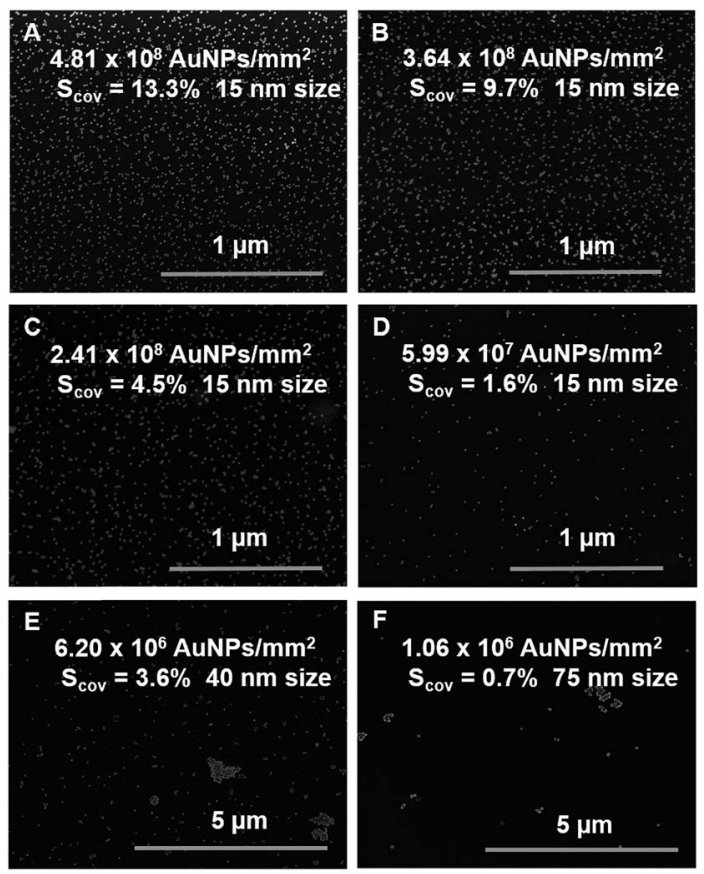

Fig. 1 SEM images of PEO-like films patterned with (A-D) decreasing number of $15 \mathrm{~nm}$ Au NPs per $\mathrm{cm}^{2}$, (E) $40 \mathrm{~nm}$ Au NPs and (F) $75 \mathrm{~nm} \mathrm{Au}$ NPs $\left(S_{\text {cov }}=\right.$ surface coverage $)$. and in an increased inter-particle distance. The number of $\mathrm{Au}$ NPs determined by SEM image analysis on $1 \mathrm{~mm}^{2}$ diameter spot for the highest concentration of $15 \mathrm{~nm}$ Au NPs (0.5 mM) was approximately $4.81 \times 10^{8} \mathrm{Au} \mathrm{NPs} / \mathrm{mm}^{2}$ whereas $3.64 \times 10^{8}$, $2.41 \times 10^{8}$ and $5.99 \times 10^{7} \mathrm{Au} \mathrm{NPs} / \mathrm{mm}^{2}$ were estimated for 0.25 , 0.125 and $0.063 \mathrm{mM}$ dispersions, respectively. The mean size of the $\mathrm{Au}$ NPs measured by SEM was $16 \mathrm{~nm}$ and the surface coverage for each condition was then respectively $13.3 \%, 9.7 \%$, $4.5 \%$ and $1.6 \%$ (Fig. 1A-D). The average inter-particle distances gradually increased when decreasing the number of nanoparticles per spot and were respectively on average $36 \pm 4 \mathrm{~nm}$, $46 \pm 3 \mathrm{~nm}, 75 \pm 10 \mathrm{~nm}$ and $112 \pm 22 \mathrm{~nm}$. In addition, spots of $40 \mathrm{~nm}$ and $75 \mathrm{~nm}$ Au NPs at a concentration of $0.5 \mathrm{mM}$ were also examined by SEM (Fig. 1E and F) and compared to the $15 \mathrm{~nm} \mathrm{Au}$ NPs spot at $0.5 \mathrm{mM}$. These experiments were performed at a given concentration i.e. same gold content $(0.5 \mathrm{mM})$, but at decreasing number of particles (due to their size increase) which resulted in a decrease of the surface coverage $(13.3 \%$ for $15 \mathrm{~nm}, 3.6 \%$ for $40 \mathrm{~nm}$ and $0.7 \%$ for $75 \mathrm{~nm})$. The measured inter-particle distance for the $40 \mathrm{~nm}$ and the $75 \mathrm{~nm}$ Au NPs were respectively: $415 \pm 87 \mathrm{~nm}$ and $771 \pm 255 \mathrm{~nm}$. The presence of aggregates of larger size $\mathrm{Au}$ NPs (40 nm and $75 \mathrm{~nm}$ ) was also observed.

The controlled average inter-particle distance and Au NPs surface distribution density suggests that attractive forces are driving the monolayer formation, following the Random Sequential Adsorption (RSA) model. ${ }^{13}$ According to the extended DLVO model (Derjaguin and Landau, Verwey and Overbeek), ${ }^{\mathbf{1 4 - 1 6}}$ the attractive long-range forces might be electrostatic forces (if the nanoparticles and the surface have opposite charges) or acid-base interaction forces (due to the interaction of polar groups in water). As the zeta-potential of the Au NPs and the surface were both negative (see ESI for details $\dagger$ ), the attractive forces could be attributed mainly to the acid-base and Lifshitz van der Waals interactions. The stability of immobilized $\mathrm{Au}$ NPs is not affected by $\mathrm{pH}$ and salt content variations suggesting that the nanoparticle-surface interaction mechanism is related to some peculiar surface properties of the PEO-like coating. In order to indirectly assess this, the PEO-like surface was irradiated by high-energy electron beam $(10 \mathrm{keV}$, at two

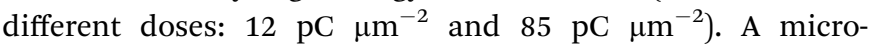
patterned surface consisting of a $40 \mu \mathrm{m}$-wide electron beam-
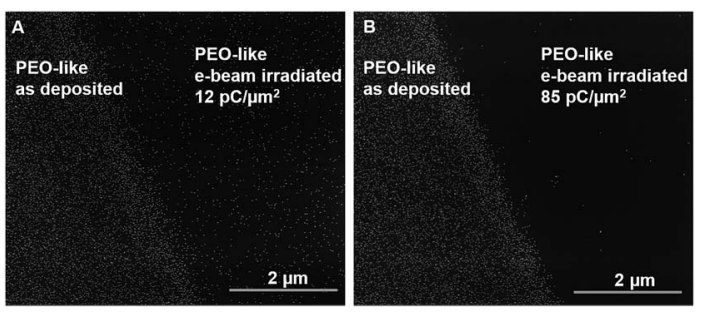

Fig. 2 SEM images of (A) Au NPs (citrate stabilized, $15 \mathrm{~nm}, 0.5 \mathrm{mM}$ ) adsorbed on the interface between the as-deposited PEO-like coating and the same coating treated by e-beam with a dose of $12 \mathrm{pC} \mu \mathrm{m}^{-2}$, (B) Au NPs (citrate stabilized, $15 \mathrm{~nm}, 0.5 \mathrm{mM}$ ) absorbed on the interface between the as-deposited PEO-like coating and the same coating treated by e-beam with a dose of $85 \mathrm{pC} \mu \mathrm{m}^{-2}$. 
treated PEO-like line surrounded by the as-deposited PEO-like coating was produced and exposed to Au NPs (15 nm, $0.5 \mathrm{mM}$ ) (Fig. 2).

The interface between the untreated PEO-like area and the

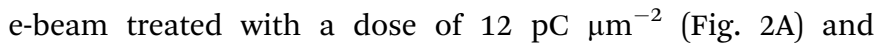

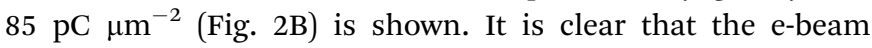
treatment prevents the adsorption of the Au NPs on the PEOlike coatings, the surface coverage decreases from $13.3 \%$ on the as-deposited area to $1.8 \%$ for the $12 \mathrm{pC} \mu \mathrm{m}^{-2}$ e-beam irra-

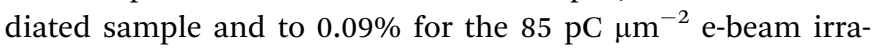
diated sample.

Our previous work ${ }^{17}$ showed that the main effect of the e-beam treatment on PEO-like coatings is the increase in refractive index of the film, which indicates an increase of the crosslinking between the polymeric chains.

Together with this effect we observed a decrease of the PEOlike character of the film, which results in a decrease of its antiadhesion properties. Since, the surface energy components (see ESI $\dagger$ ) do not change dramatically, the increase of the film crosslinking which reduces the mobility of the PEO-like chains in water i.e. lowers the "contact area" with the nanoparticles is the possible cause of the decrease of Au NPs adsorption to the surface.

These results clearly demonstrate that the surface physicochemical properties such as the chemical composition and mechanical properties of the PEO-like coating are the key parameters favoring the controlled deposition of the citrate stabilized Au NPs.

We can conclude that, as expected, the Au NPs are attracted to the surface by the acid-base and Lifshitz van der Waals interaction forces, which are overtaking the electrostatic repulsion forces. The attractive forces determine a RSA-like formation of the Au NPs monolayer, enabling the creation of controlled nanocomposites between the as-deposited PEO-like surface and the Au nanostructures. Experiments in different conditions confirmed the extreme stability of the Au NPs once adsorbed onto the PEO-like coating. In the $\mathrm{pH}$ range 2-11, the $\mathrm{Au}$ NPs binding was stable for weeks. This also confirmed that electrostatic interactions were not driving the Au NPs adsorption, since drastic $\mathrm{pH}$ changes would lead to the detachment of the particles from the surface.

\section{Functionalized Au NPs by self-assembled monolayers (SAMs) for specific detection using QCM-D in flow mode}

The irreversible attachment of Au NPs chemical bio-activity on PEO-like coatings has been also demonstrated by monitoring of their absorption using a Quartz Crystal Microbalance with Dissipation Monitoring (QCM-D) technique. A citrate-stabilized dispersion of $15 \mathrm{~nm} \mathrm{Au}$ NPs at a concentration of $0.5 \mathrm{mM}$ was injected into the system at a flow rate of $10 \mu \mathrm{min}^{-1}$ on two PEO-like coated quartz chips (noted Q1 and Q2). The measured frequency shifts, $\Delta F$, and dissipation shifts, $\Delta D$, are shown in Fig. 3 as a function of time.

After reaching saturation of the signal of adsorption on both quartz chips, a rinsing step was successively performed. The washing operation induced almost no changes in the measured

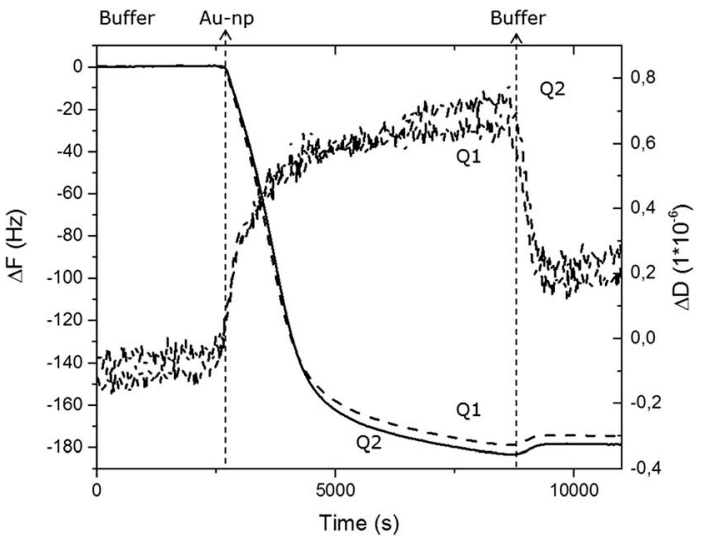

Fig. 3 QCM-D monitoring of the adsorption of citrate-stabilized 15 $\mathrm{nm}$ Au NPs (0.5 mM, pH =6.5) on PEO-like coated samples (Q1 and Q2). $\Delta F$ represents the frequency shift and $\Delta D$, the dissipation shift.

Table 1 Compilation of experimental and calculated data for the adsorption of Au NPs on PEO-like coatings (Q1 and Q2) ${ }^{a}$

\begin{tabular}{lll}
\hline Au NPs $15 \mathrm{~nm}$ on PEO & $\mathrm{Q} 1$ & $\mathrm{Q} 2$ \\
\hline$\Delta F(\mathrm{~Hz})$ & -180 & -175 \\
$\Delta m(\mathrm{ng})$ & 637.2 & 619.5 \\
$N_{\text {AuNP }}($ particles $)$ & $1.87 \times 10^{10}$ & $1.82 \times 10^{10}$ \\
$S_{\text {AuNP }}\left(\mathrm{cm}^{2}\right)$ & $1.32 \times 10^{-1}$ & $1.28 \times 10^{-1}$ \\
$S_{\mathrm{COV}}$ & $19 \%$ & $19 \%$
\end{tabular}

${ }^{a} \Delta F$ represents the frequency shift and $\Delta m$, the adsorbed mass. $N_{\mathrm{AuNP}}$ is the total number of Au NPs bound on the surface, $S_{\mathrm{AuNP}}$ is the total surface of Au NPs in $\mathrm{cm}^{2}$ and $S_{\mathrm{COV}}$ represents the surface coverage of $\mathrm{Au}$ NPs in percentage of the total area available for binding of the nanoparticles.

frequency confirming that the adsorption of Au NPs is irreversible under these experimental conditions. The relatively small dissipation value $(\Delta D)$ obtained at saturation indicates that the Au NPs are rigidly bound to the PEO-like layer, permitting the use of the Sauerbrey approximation to calculate the corresponding adsorbed mass from the measured value of the frequency shift $(\Delta F)$ (Table 1). The adsorbed mass of Au NPs on sample Q1 and Q2 were respectively $637 \mathrm{ng}$ and $620 \mathrm{ng}$, corresponding to the total number of $1.87 \times 10^{10}$ and $1.82 \times$ $10^{10} \mathrm{Au}$ NPs adsorbed respectively on Q1 and Q2. The surface coverage of Au NPs obtained in flow conditions was found to be slightly higher $(19 \%)$ than in static conditions (13.3\%). The similarity of the results obtained for both PEO-like coated samples confirmed the reproducibility of the absorption of $\mathrm{Au}$ NPs on PEO-like surfaces.

After the stable adsorption of the Au NPs monolayer, the immobilized Au nanostructures were selectively functionalized by thiolated alkyl chains, terminated with $-\mathrm{COOH}$ functional groups to form Self-Assembled Monolayers (SAMs) on the Au NPs surface via sulphur bonds. The self-assembling process of the SAMs was carried out overnight in order to allow the formation and the ordered arrangement of the monolayer. The same procedure was applied to a PEO-like coated QCM-D quartz 


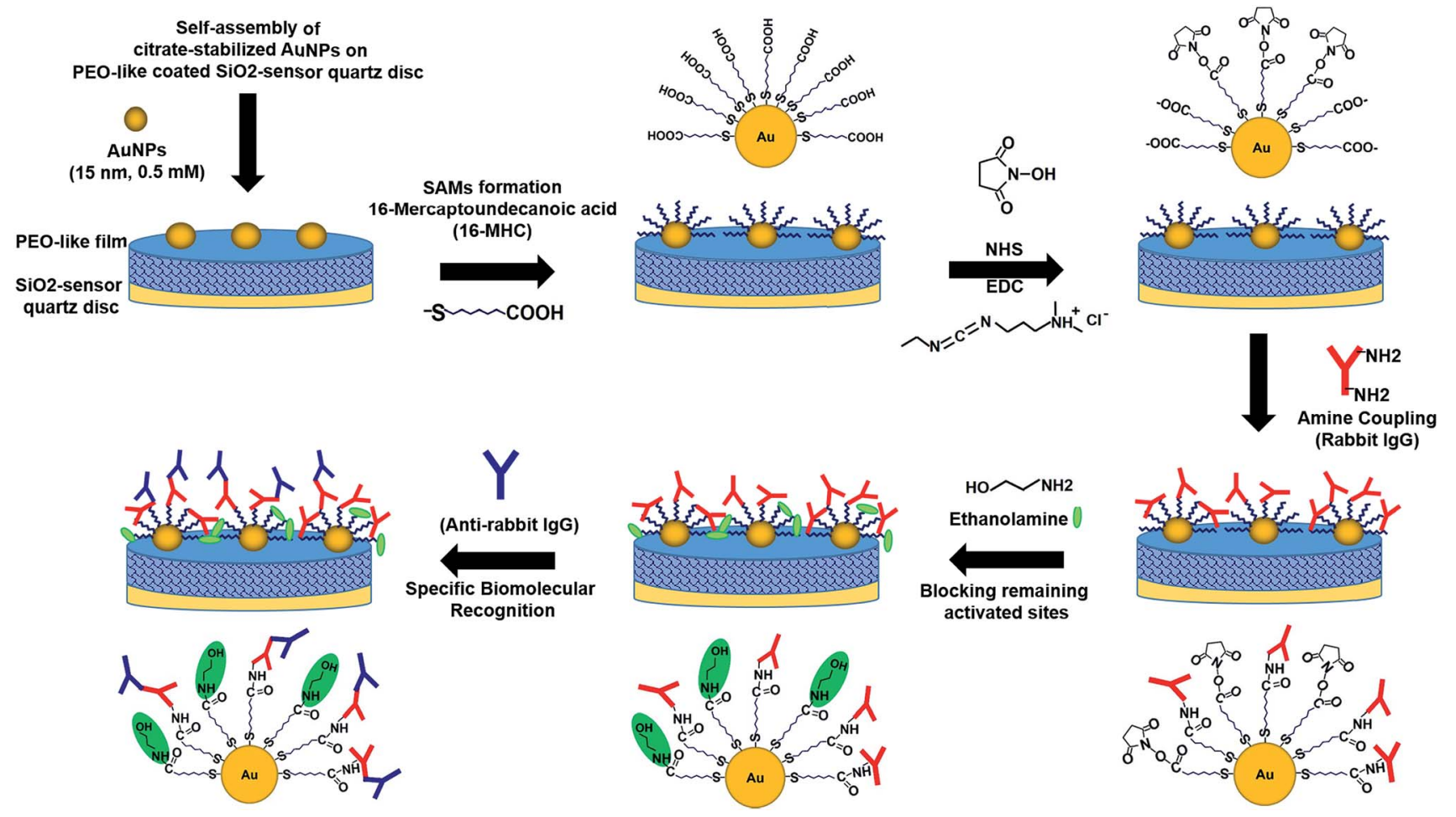

Fig. 4 Schematic representation of the successive steps occurring during the immobilization of Au NPs on PEO-like coated QCM quartz, the online surface functionalization and immunodetection.

chip without particles (Q3) and to a flat gold-coated QCM-D chip (Q4) that are used as controls. Then, the QCM-D chips were rinsed and dried by ultrapure nitrogen stream. The four chips (two chips with Au NPs on PEO-like coating (Q1 and Q2), one chip with PEO-like coating (Q3), and one chip with standard flat gold (Q4)) were then inserted in the instrument for real-time monitoring of protein binding experiments. The $-\mathrm{COOH}$ terminal groups of the SAMs were activated using the standard EDC/NHS protocol that allowed the covalent binding of rabbit IgGs through their primary amine groups. The unreacted $-\mathrm{COOH}$ groups were then inactivated by ethanolamine solution. The following step consisted in the measurement of the specific recognition of the covalently immobilized rabbit IgG by polyclonal anti-rabbit IgG. As a negative control, an anti-FLAG antibody was flown on one of the two chips with Au NPs on PEO-like film (Q2). Each step was followed by rinsing with the buffer in order to eliminate the non-covalently bound molecules. The step-by-step procedure is presented schematically in Fig. 4.

The whole experiment was performed in parallel on the four QCM-D chips and the variation of the resonance frequency $(\Delta F)$ of the quartz and the variation of the dissipation factor $(\Delta D)$ were monitored in real time. The resulting time-dependent QCM-D binding curves are presented in Fig. 5.

On samples Q1 and Q2, the injection of IgG resulted in the negative variation of the resonance frequency and a contemporary positive variation of the dissipation component as a consequence of the binding of the IgGs to the surface. The rinsing step shows that IgG remain stably bound to the surface, since no dissociation is evidenced in the kinetic curves. The amount of bound IgG was calculated using the Sauerbrey equation. Frequency shifts measured on Q1 and Q2 quartz chips correspond to respectively 250 and $268 \mathrm{ng}$. Remarkably, approximately the same amount of proteins binding (240 $\mathrm{ng}$ ) has been measured on the flat gold chip Q4 despite the fact that this chip presents a total gold surface available for binding of almost the double compared to the ones obtained on the chips modified with Au NPs $\left(0.2 \mathrm{~cm}^{2}\right.$ instead of around $\left.0.13 \mathrm{~cm}^{2}\right)$. On the PEO-like coated QCM-D chip (Q3), a much lower amount of IgG was immobilized (around $40 \mathrm{ng}$ ) confirming the protein adhesion resistant properties of PEO-like films. The low protein binding signals on PEO-like films are most likely due to the presence of some impurities from the plasma deposition phase that are activated by the EDC/NHS molecules and that are able to covalently bind the proteins. If we compare the amount of IgG molecules immobilized on the Q4 (flat gold surface), we can estimate that these "grafted" active groups on the PEO-like film represent only the $16 \%$ of the total surface. Consistently, the $84 \%$ of the surface is retaining its anti-adhesive property. Nevertheless, these results show that the amount of IgG immobilized on Q1 and Q2 are mostly located on the Au NPs. Considering the $19 \%$ of surface coverage of the Au NPs on the PEO-like coating, the surface density $\left(\sigma_{\mathrm{IgG}(\mathrm{AuNP})}\right)$ of IgGs on the $\mathrm{Au}$ NPs can be determined through the equation:

$$
\begin{aligned}
\sigma_{\mathrm{IgG}(\mathrm{AuNP})} & =\left[m_{\mathrm{IgG}(\mathrm{TOT})}-m_{\mathrm{IgG}(\mathrm{PEO})} \times 0.81\right] / S_{\mathrm{AuNP}} \\
& =(250.0-34.5) / 1.32 \mathrm{ng} \mathrm{cm}^{-2}=163 \mathrm{ng} \mathrm{cm}^{-2}
\end{aligned}
$$



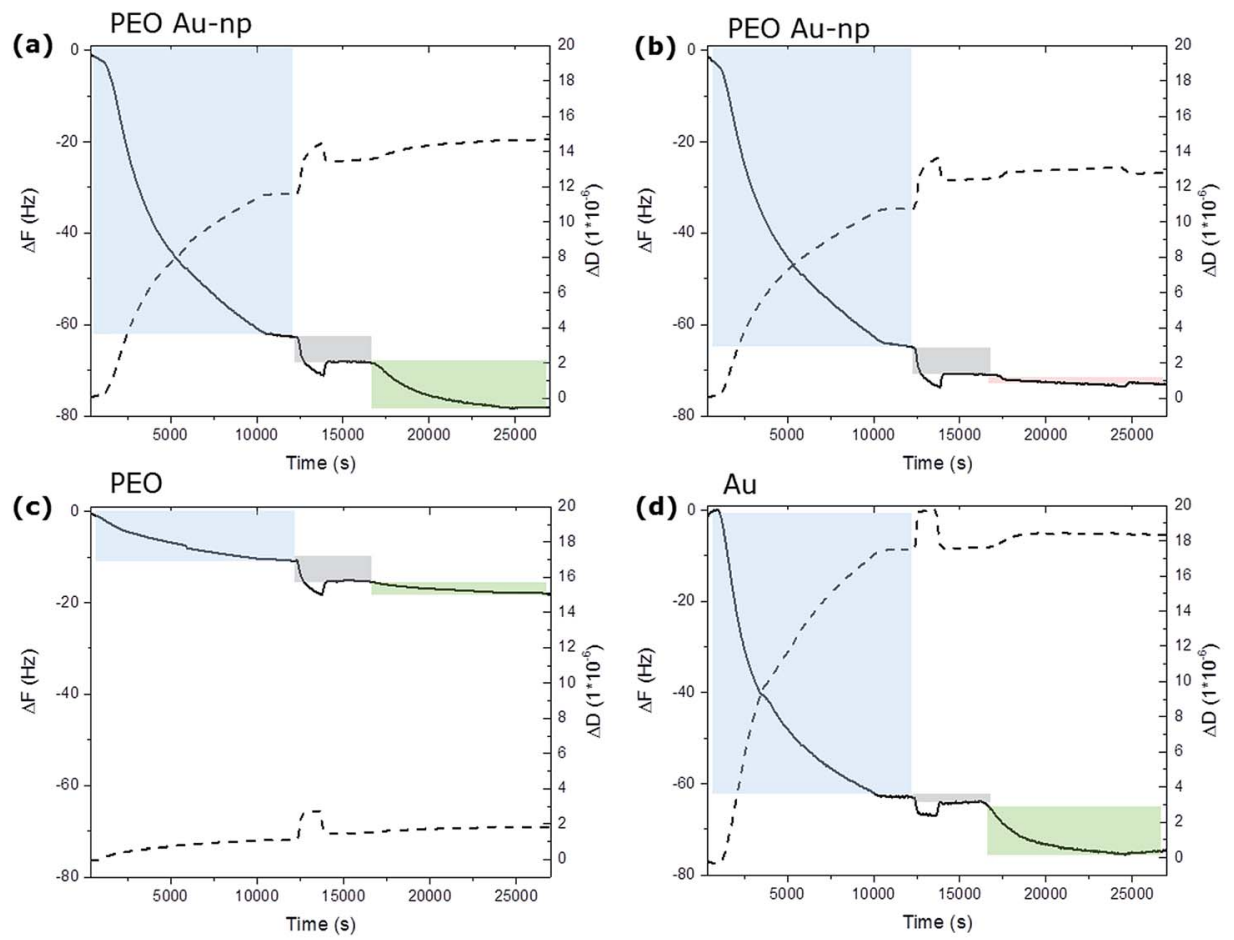

(e)

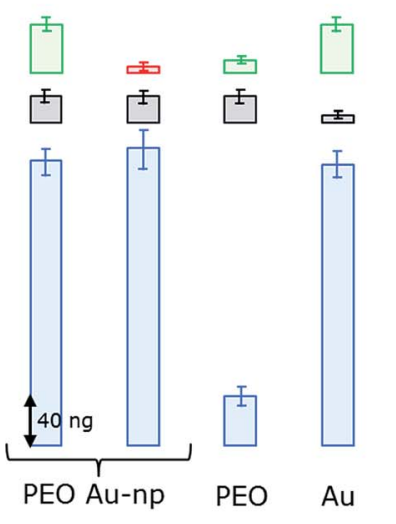

Fig.5 QCM results showing the online functionalization of Au NPs nanostructured PEO-like coated samples (a and b), PEO-like coating only (c) and flat gold (d) samples with rabbit IgGs, followed by specific recognition with anti-rabbit IgGs on Q1, Q3 and Q4. A solution of anti-FLAG antibodies was injected on sample $Q 2$ as a negative control. $\Delta F$ represents the frequency shift and $\Delta D$, the variation of the dissipation frequency. The immobilized mass for each step was calculated using the Sauerbrey equation and reported in (e).

where $m$, is the adsorbed mass and $S$, is the total surface of $\mathrm{Au}$ NPs.

On the other hand, the same calculation on Q4 gives a surface density of:

$$
\sigma_{\mathrm{IgG}(\mathrm{Au})}=\left[m_{\mathrm{IgG}(\mathrm{Au})}\right] / S_{\mathrm{Au}}=(240) / 2 \mathrm{ng} \mathrm{cm}^{-2}=120 \mathrm{ng} \mathrm{cm}^{-2}
$$

This shows that the surface density of IgG is around $25 \%$ larger on the Au NPs than on the corresponding gold flat surface. Moreover, the difference in the dissipation factor $(\Delta D)$ $\left(12 \times 10^{-6}\right.$ on the Au NPs and $20 \times 10^{-6}$ on the flat gold surface $)$ together with the higher surface density calculated from the frequency shift on the Au NPs compared to the flat gold suggests that the Au NPs induce the formation of a more dense and more tightly bound layer of proteins on a gold nanoparticle than on a flat surface.

After the IgG binding experiments, the unreacted $-\mathrm{COOH}$ groups of the SAMs were de-activated by ethanolamine $(200 \mathrm{mM})$. It is possible to notice that some ethanolamine molecules are absorbed by the PEO (evidenced by the grey bars in Fig. 5, Q1, Q2, Q3), while the adsorption is negligible on the flat gold chip (Q4). Then, the anti-IgG antibodies were flown on Q1, Q3 and Q4 while the anti-FLAG antibodies (the negative control) were flown on Q2. The results show that approximately $50 \mathrm{ng}$ of anti-IgG on Q1 and Q4, and $8 \mathrm{ng}$ on Q3, while less than $5 \mathrm{ng}$ of anti-FLAG were detected on Q2. These results clearly demonstrate that only a part of the covalently immobilized IgG kept its structural conformation and enable the specific recognition by anti-IgG antibodies. The specificity is confirmed by the very low unspecific binding detected on Q2. Moreover, these results show that the IgG immobilized on the Au NPs have comparable activity than the ones immobilized on the flat gold surface. In fact, dividing the amount of specifically bound antiIgG on the Au NPs (calculated by subtracting to the absorbed mass of IgG, the mass of the anti-FLAG and subtracting the contribution of the PEO) by the number of the IgG (subtracting the contribution of the PEO) one obtains that $17 \%$ of the immobilized IgG were recognized by their antibody. The same calculation gives a value of $18 \%$ of activity for flat gold. These results show that the Au NPs nanostructured PEO-like coated surface presents a similar biomolecular activity with the flat gold surface, showing the suitability of such nanostructured surfaces for controlling the immobilization of biomolecules with nanoscale resolution.

\section{Patterning of Au NPs domains on PEO-like coatings for the creation of cell arrays}

Cell and protein micropatterning have important applications in the development of detection platforms, tissue engineering and fundamental cell biology studies. ${ }^{18}$ Hereby, experiments have been performed to assess the suitability of our Au nanostructured surface to modulate the surface bio-functionality for further cell adhesion studies. Cell culture experiments have been performed on the Au NPs nanostructured surfaces to evaluate the cell adhesive properties of Au NPs versus their surface coverage. For that purpose, A549 human epithelial cells 


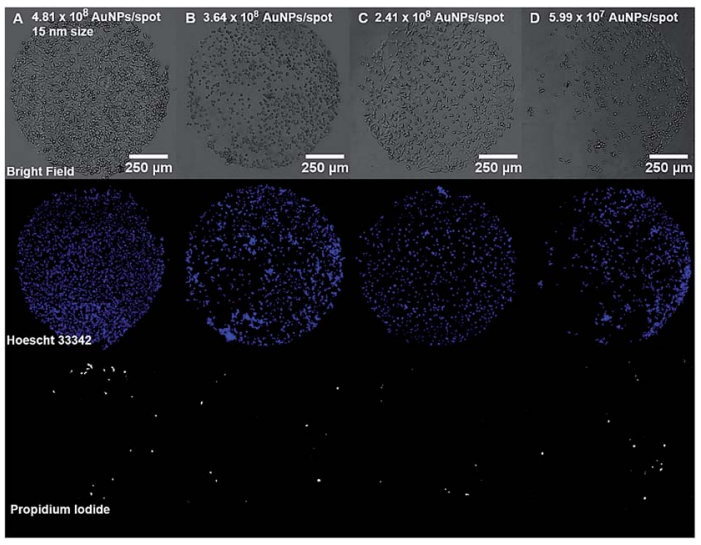

Fig. 6 Top line corresponds to bright field images of A549 cells attachment on $15 \mathrm{~nm}$ AuNP patterns of concentration (A) $0.5 \mathrm{mM}$, (B) $0.25 \mathrm{mM}$, (C) $0.125 \mathrm{mM}$, (D) $0.063 \mathrm{mM}$. Middle and lower lines shows the corresponding viability of cells for each condition (Hoescht 33342 is staining all cell nuclei and $\mathrm{PI}$ is staining selectively the necrotic cell nuclei).

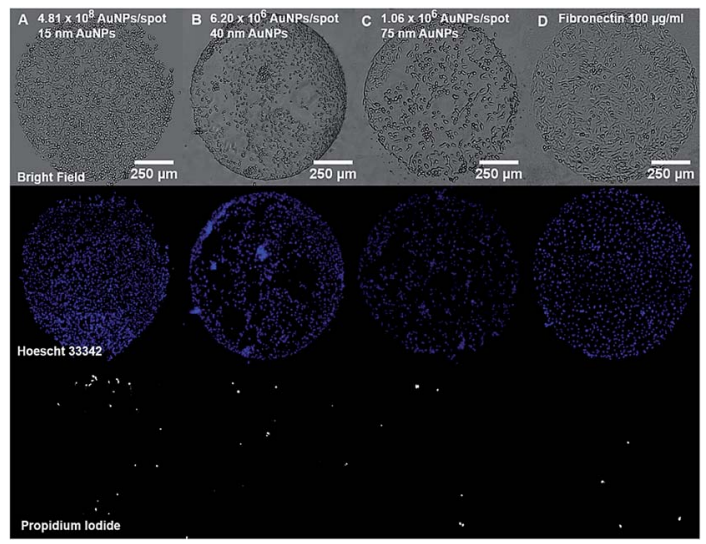

Fig. 7 Top line corresponds to bright field images of A549 cells attached on Au NPs patterns of $0.5 \mathrm{mM}$ concentration of (A) $15 \mathrm{~nm}$, (B) $40 \mathrm{~nm}$, (C) $75 \mathrm{~nm}$. Middle and lower lines shows the corresponding viability of cells for each condition (Hoescht 33342 is staining all cell nuclei and $\mathrm{Pl}$ is staining selectively the necrotic cell nuclei). Scale bar = $250 \mu \mathrm{m}$.

were seeded on glass slides containing four spots of decreasing concentrations of $15 \mathrm{~nm}$ Au NPs $(0.5,0.25,0.125,0.063 \mathrm{mM})$ immobilized on a PEO-like coating. Bright field images taken after $24 \mathrm{~h}$ incubation of cells on Au NPs spots showed the attachment of cells on gold nanopatterns only, while PEO-like coated areas remained cell-repellent (Fig. 6 and 7).

As expected, decreasing the number of nanoparticles per spot reduced the number of adhesion points and thus the overall attachment of cells (Fig. 6). ${ }^{6}$ The number of cells attached per spot (size $1 \mathrm{~mm}^{2}$ ), estimated by counting the number of Hoechst 33342 positive cell nuclei, was approximately 1800 for $0.5 \mathrm{mM}, 1100$ for $0.25 \mathrm{mM}, 850$ for $0.125 \mathrm{mM}$ and 750 cells for $0.06 \mathrm{mM} \mathrm{Au}$ NPs spots.

The attachment of A549 cells was also observed on spots of 40 and $75 \mathrm{~nm} \mathrm{Au} \mathrm{NPs}$ at their highest concentration $(0.5 \mathrm{mM})$
(Fig. 7). The number of cells attached was respectively around 1500 and 770 cells per spot. However, cells were poorly distributed due to Au NPs aggregates formation and decreased number of anchoring points on the surface, as shown on previous SEM images (Fig. 1E and F).

Prior investigations by our group showed that an extracellular matrix protein, fibronectin, adsorbed on PEO-like coatings can be successfully used for creating cell adhesive domains. ${ }^{\mathbf{1 1 , 1 2}}$ Therefore, the attachment of A549 cells on fibronectin spots was also studied here for comparison with the Au NPs spots. Morphological analysis showed that A549 cells were better attached and spread on the fibronectin spot than on the Au NPs patterns. This difference can be explained by the fact that fibronectin is a natural component of the cell's microenvironment and therefore is more attractive than Au NPs, especially on a repellent substrate.

Viability of A549 cells on Au NPs patterns was assessed using propidium iodide (PI) and Hoechst 33342 staining. As seen in Fig. 6 and 7, most cells residing on the spots were alive, while only few PI-positive, necrotic cells could be observed, and their number was comparable to the one on the fibronectin spot, suggesting that Au NPs patterns are biocompatible.

In order to further improve the biocompatibility of $\mathrm{Au}$ nanopatterned surface we functionalized Au NPs with fibronectin (Fn) by direct electrostatic immobilization. The specific conditions at which Fn was immobilized on Au NPs and not on the PEO-like background were previously studied and in particular the role of the $\mathrm{pH}$ of the Fn solution during the immobilization. As indicated in our previous works, ${ }^{11,12}$ Fn can be successfully immobilized on PEO-like films by working at the isoelectric point of the protein $(\mathrm{pH}=5.5)$. On the other hand, by working at a slightly more basic $\mathrm{pH}(\mathrm{pH}=7.4)$, the electrostatic

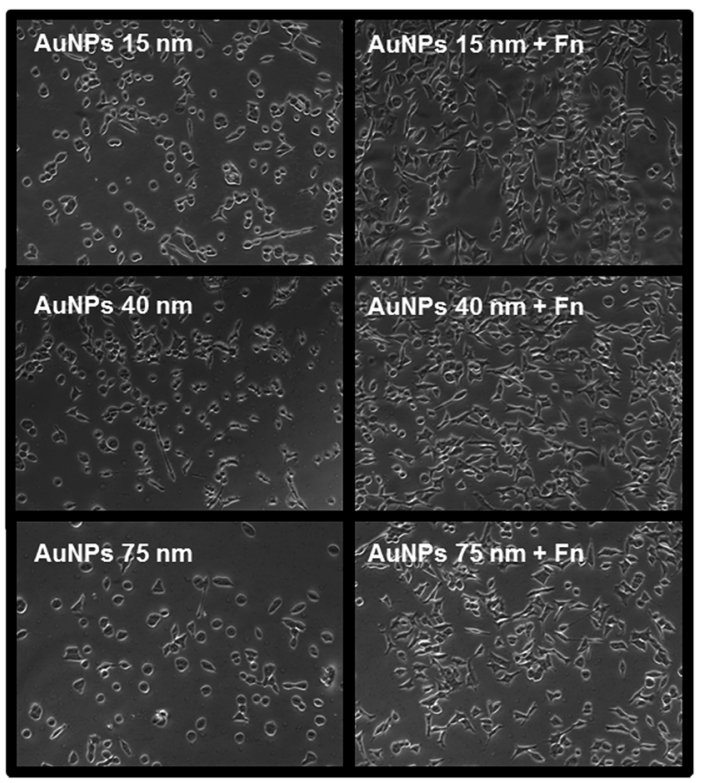

Fig. 8 Bright-field images for the A549 cells cultured on PEO-like Au NPs nanopatterns without (left column) and with (right column) fibronectin functionalization. For the Au NPs of the three different sizes (from top $15 \mathrm{~nm}, 40 \mathrm{~nm}$ and $75 \mathrm{~nm}$ ). 
repulsion is more effective and only a negligible amount of Fn is immobilized on the PEO-like films, as evaluated by QCM-D measurement (the measured $\Delta F$ at $\mathrm{pH} 7.4$ of Fn was $\approx 0 \mathrm{~Hz}$, corresponding to a negligible mass of Fn). This result was confirmed by the absence of cell adhesion on the PEO-like coating functionalized with $\mathrm{Fn}$ at $\mathrm{pH}=7.4$.

In the second step Au NPs nanopatterns on PEO were exposed to Fn solution in the same conditions (i.e. at $\mathrm{pH}=7.4$ ), in order to selectively functionalize the spots of Au NPs. The maximum surface coverage for each Au NPs size was used for these experiments. Bright field microscopy images show that the Fnfunctionalized Au NPs promoted cell adhesion and spreading with a higher efficiency as compared to the "naked" Au NPs (Fig. 8). On Fn-functionalized areas cells were able to reach higher confluence and displayed more physiological morphology comparing to non-functionalized areas, where we observed many round cells, indicating reduced cell affinity to the surface.

\section{Conclusions}

In summary, we have demonstrated that PEO-like coated surfaces can be utilized as a simple and robust platform for creating nanostructured bio-interfaces. Two-dimensional patterns of a single layer of particles on the micrometer-tosubmicron scale with controllable inter-particle distance average have been generated. We have shown that the deposition of citrate-stabilized Au NPs on PEO-like coatings is fast, direct and irreversible due to a combination of electrostatic and hydrophobic forces. By the tuning of the surface concentration of $\mathrm{Au}$ NPs and of their size, it was possible to control the surface density of Au nanostructures.

The gold nanostructured surface was shown to be cell adhesive and biocompatible. The number of cells adhering to the Au NPs spots was proportional to the NPs density on the PEO-like surface. Such spatial and temporal control of cell adherence is especially important for the development of cellbased biosensors designed to monitor physiological changes in cells upon stimulation. ${ }^{19}$ Furthermore, we demonstrated that the Au nanostructures can be selectively functionalized by selfassembled monolayers without affecting the non-adhesive properties of the PEO-like coating background. The surface becomes then an ideal platform to study protein-nanoparticles and cell-nanoparticles interactions using affinity-based biosensors, like QCM, since it is possible to discriminate the signal related to the nanoparticles alone.

These results show the capability to immobilize proteins with a controlled spatial distribution and density, which enables the modulation at nanoscale of the bio functionality of the surface. This platform is therefore a good candidate for designing multifunctional nanostructured interfaces for nanoengineering and nano-bioelectronics applications.

\section{Methods}

\section{Preparation of substrates}

Double-sided polished silicon wafers (from Institute of Electronics Material Technology) were used for surface analysis by
XPS, ellipsometry and SEM imaging. Standard optical glass slides (Thermo Scientific Menzel-Glaser) were used for cell culture experiments and immunofluorescence. They were cut in $1 \mathrm{~cm} \times 1 \mathrm{~cm}$ squares, cleaned by sonication in $70 \%$ ethanol for 5 min and dried with a nitrogen flush gun. $\mathrm{SiO}_{2}$-coated sensor quartz discs (14 $\mathrm{mm}$ in diameter, $0.30 \mathrm{~mm}$ thick) with a fundamental frequency of $5 \mathrm{MHz}$ (Q-sense $\mathrm{AB}$, Gothenburg, Sweden) were used in QCM-D experiments. The quartz crystals were cleaned by immersion in $70 \%$ ethanol and dried with a nitrogen flush gun. For surface zeta potential measurements, glass microslides $(76 \mathrm{~mm} \times 26 \mathrm{~mm}$, Thermo Scientific MenzelGlaser) were cleaned by sonication in $70 \%$ ethanol for $5 \mathrm{~min}$ and dried with a nitrogen flush gun.

\section{PEO-like deposition by plasma polymerization}

The plasma reactor used in this study was a home-made stainless-steel reactor (vessel size $300 \times 300 \times 150 \mathrm{~mm}^{3}$ ) with two symmetrical internal parallel-plate electrodes (diameter of electrodes: $140 \mathrm{~mm}$, distance between two electrodes: $50 \mathrm{~mm}$ ). The plasma was generated by a radio frequency (RF) generator (13.56 MHz) connected to the upper electrode, whereas the bottom electrode was grounded and used as a sample holder. Plasma polymerization was carried out in continuous plasma discharge mode with a power ranging from 20 to $22 \mathrm{~W}$ and a bias of $150 \mathrm{~V}$. The reflected power was adjusted to its minimum value ( $<1 \%$ of incident power). Pure Diethylene Glycol Dimethyl Ether (DEGDME, $\left(\mathrm{CH}_{3} \mathrm{OCH}_{2} \mathrm{CH}_{2}\right)_{2} \mathrm{O}$ ) vapors (Sigma Aldrich) was used as a gas feed for the deposition of poly(ethyleneoxide) films (hereafter PEO-like films) with a monomer initial pressure of around 15 mTorr for a final working pressure of 100 mTorr, a monomer vessel temperature of $45 \pm 0.5{ }^{\circ} \mathrm{C}$, and a gas line temperature of around $55^{\circ} \mathrm{C}$. Gas flow rates were regulated by MKS mass-flow controllers and the pressure was monitored by a MKS baratron. The working pressure in the chamber was maintained by a rotatory pump.

Between each deposition, the plasma chamber was cleaned for half an hour by pure oxygen plasma (100 mTorr with a power of $55 \mathrm{~W}$ and bias of $400 \mathrm{~V}$ ). In addition, substrates were also cleaned by oxygen plasma for $5 \mathrm{~min}$ before the deposition of PEO-like film.

Measurements using ellipsometry show that the deposition rate of the reactor is $7 \mathrm{~nm} \min ^{-1}$ for glass and silicon wafer samples. The film obtained is uniform on the substrate with the best antifouling and cell repellent properties at a thickness of 30 $\mathrm{nm}$. Prior to surface analysis, surface functionalization or biological tests, the samples were carefully washed with ultra-pure water and dried with a nitrogen flush gun to remove possible surplus of monomer and physisorbed material. Results of surface characterization (XPS, ellipsometry, contact angle and zeta-potential) of the PEO-like films can be found in the ESI. $\dagger$

\section{Scanning electron microscopy (SEM) analysis}

SEM measurements were performed by a FEI NOVA 600, Dual Beam, using $5 \mathrm{keV}$ acceleration voltage and acquiring secondary electrons. The average size of particles was calculated by Image J software, taking into account at least 100 particles. E-beam 
irradiation of the PEO-like coating has been carried out using the electron beam at $10 \mathrm{keV}$ and by controlling the dose of electrons delivered to the surface by controlling the exposure time.

\section{Gold nanoparticles synthesis}

Synthesis of 15 nm Au NPs. Synthesis of 15 nm sized Au NPs was carried out by modification of the procedure described by Turkevich et al., ${ }^{17}$ in which the gold nanoparticles are produced by the reduction of the gold salt by the sodium citrate that acts as a reducing agent and stabilizer. In this work, the solution was heated up using a specialized microwave apparatus (Discover S by CEM corporation) to ensure a highly reproducible rapid heating. In this method, $5 \mathrm{ml}$ of tetrachloroauric acid trihydrate $0.01 \mathrm{M}\left(\mathrm{HAuCl}_{4} \cdot 3 \mathrm{H}_{2} \mathrm{O}\right)$ (Sigma-Aldrich) was dissolved in $95 \mathrm{ml}$ of water. The solution was rapidly heated up and hold at $97{ }^{\circ} \mathrm{C}$ for 5 minutes using a maximum microwave power of $250 \mathrm{~W}$ under vigorous mechanical stirring. In that condition, $2.5 \mathrm{ml}$ of trisodium citrate dihydrate $0.1 \mathrm{M}$ (Sigma-Aldrich) was added to the solution and kept at $100{ }^{\circ} \mathrm{C}$ for further 20 minutes. Afterwards, the solution was rapidly cooled down to $40{ }^{\circ} \mathrm{C}$.

\section{Synthesis of $40 \mathrm{~nm} \mathrm{Au} \mathrm{NPs}$}

The synthesis of $40 \mathrm{~nm}$ Au NPs were carried out by regrowth method of $15 \mathrm{~nm}$ Au NPs. $95 \mathrm{ml}$ of MilliQ water were left to stir at $60{ }^{\circ} \mathrm{C}$ until the equilibrium is reached. Then, $2.8 \mathrm{ml}$ of sodium citrate dihydrate $(0.1 \mathrm{M})$ and $0.42 \mathrm{ml}$ of $200 \mathrm{mM}$ of sodium hydroxide (Sigma-Aldrich) were added to the water solution. After 30 minutes, $2.24 \mathrm{ml}$ of $\mathrm{HAuCl}_{4} \cdot 3 \mathrm{H}_{2} \mathrm{O} 10 \mathrm{mM}$ and $2.6 \mathrm{ml}$ of $12 \mathrm{~nm}$ gold nanoparticles were added to the solution, under vigorous stirring. The solution was left to react for $48 \mathrm{~h}$ at $60{ }^{\circ} \mathrm{C}$. The nominal concentration of gold in $\mathrm{Au}$ NPs was $0.24 \mathrm{mM}$.

\section{Synthesis of $75 \mathrm{~nm}$ Au NPs}

The synthesis of $75 \mathrm{~nm}$ Au NPs were carried out by regrowth method of $40 \mathrm{~nm}$ Au NPs. $70 \mathrm{ml}$ of MilliQ water were left to stir at $60{ }^{\circ} \mathrm{C}$ and then $2.8 \mathrm{ml}$ of sodium citrate dihydrate $(0.1 \mathrm{M})$ and $0.42 \mathrm{ml}$ of $200 \mathrm{mM}$ of sodium hydroxide (Sigma-Aldrich) were added to the water solution. After 30 minutes, $1.25 \mathrm{ml}$ of $\mathrm{HAuCl}_{4} \cdot 3 \mathrm{H}_{2} \mathrm{O} 10 \mathrm{mM}$ and $25 \mathrm{ml}$ of $40 \mathrm{~nm}$ Au NPs were added to the solution, under vigorous stirring. The reaction time was $48 \mathrm{~h}$ at $60{ }^{\circ} \mathrm{C}$. The nominal concentration of gold in Au NPs was $0.17 \mathrm{mM}$.

The Au NPs were characterized by Dynamic Light Scattering (DLS), Centrifugal Liquid Sedimentation (CLS) and Scanning Electron Microscopy SEM (see ESI for details $\dagger$ ).

\section{Quartz crystal microbalance with dissipation monitoring (QCM-D) analysis}

The immobilization of $15 \mathrm{~nm}$ Au NPs on a plasma deposited PEO-like coating (30 nm thickness) was investigated utilizing the QCM-D technique, by monitoring simultaneously frequency and dissipation changes in real time. A small mass added to the sensors $(\Delta m)$ induces a decrease in resonant frequency $(\Delta f)$, which is proportional to $\Delta m$, provided the mass is evenly distributed and is sufficiently rigid and/or thin to have negligible internal friction: $\Delta m=-C \times \Delta f / n$ where $C(=17.7$ $\mathrm{ng} \mathrm{cm} \mathrm{cm}^{-2}$ at $f=5 \mathrm{MHz}$ ) is the mass-sensitivity constant and $n(=1,3,5 \ldots)$ is the overtone number. The instrument was a QCM-D E4 (Q-Sense AB, Gothenburg, Sweden), which uses four temperature-stabilized measurement cells (in parallel configuration in our case).

A total of three individual PEO-like coated sensor quartz discs (Q-Sense AB, Gothenburg, Sweden, nominal resonant frequency of $5 \mathrm{MHz}$ ) were assembled into the QCM and were first exposed to ultrapure water for at least one hour in order to stabilize the system. In addition, a fourth uncoated sensor quartz disc was used as a negative control. Then, a solution of $2.5 \mathrm{mM}$ of citrate buffer ( $\mathrm{pH}$ 6.5) was run on all four samples in order to eliminate any possible buffer change response. Once stable, a solution of $15 \mathrm{~nm} \mathrm{Au} \mathrm{NPs} \mathrm{was} \mathrm{pumped} \mathrm{through} \mathrm{the}$ measurement chamber only on the first two PEO-like coated sensor quartz disc (Q1 and Q2) by applying a flow rate of $10 \mu \mathrm{l}$ $\min ^{-1}$. The third PEO-like coated (Q3) and the fourth uncoated quartz (Q4) were used later as negative control. The temperature was stabilized at $25{ }^{\circ} \mathrm{C}$ throughout the experiment. The frequency and dissipation changes due to the adsorption of $\mathrm{Au}$ NPs were monitored until stable signals were recorded at saturation. Once the two PEO-coated quartz crystals were saturated with Au NPs, all four samples were firstly functionalized overnight in with $1.44 \mathrm{mg} \mathrm{ml}^{-1}$ of 16-mercaptohexadecanoic acid in ethanol (16-MHC, Sigma-Aldrich) to form a self-assembled monolayer. The next day, samples were rinsed with $70 \%$ ethanol and dried carefully. The carboxylic groups of the SAM were then activated using a mixture of $N$-hydroxysuccinimide (NHS, 0.1 M in $\mathrm{H}_{2} \mathrm{O}$, Sigma Aldrich) and ethyl(dimethylaminopropyl) carbodiimide (EDC, $0.4 \mathrm{M}$ in $\mathrm{H}_{2} \mathrm{O}$, Sigma Aldrich) for 20 minutes at room temperature. Samples were then rinsed with water and dried carefully before being assembled into the QCM. All four samples were then functionalized with a solution of rabbit IgG anti-TLR2 (Santa Cruz, H175 sc-10739) at a concentration of $5 \mu \mathrm{g} \mathrm{ml}^{-1}$ for 3 hours at a flow rate of $10 \mu \mathrm{l} \mathrm{min}^{-1}$. Subsequently, surfaces were firstly rinsed with PBS and then deactivated and blocked with $200 \mathrm{mM}$ ethanolamine (Sigma-Aldrich) for one hour. After functionalization and blocking of the surface, a solution of $5 \mu \mathrm{g} \mathrm{ml} \mathrm{m}^{-1}$ of goat anti-rabbit IgG-Texas red (Santa Cruz, sc2780) was injected on Q1, Q3 and Q4 for 3 hours. A solution of $5 \mu \mathrm{g} \mathrm{ml}^{-1}$ mouse anti-FLAG (Sigma-Aldrich, F1804) was also injected as a negative control on sample Q2 to demonstrate specificity. The variation in frequency proportional to a specific biorecognition was followed in real time. Ultimately, the running buffer was flown on all four samples to wash away unbound biomolecules.

\section{Patterning of Au NPs spots on PEO-like coatings}

Serial dilutions of Au NPs dispersion $(0.5,0.25,0.125,0.06 \mathrm{mM})$ of different sizes $(15,40$ and $75 \mathrm{~nm}$ ) were prepared in $2.5 \mathrm{mM}$ citrate buffer (pH 6.5). Fibronectin (Sigma-Aldrich, F0895) was also used as a control to create spots of cell adhesive domains 
on a cell repellent PEO-like coated film. Fibronectin was diluted to a concentration of $100 \mu \mathrm{g} \mathrm{ml}^{-1}$ in printing buffer consisting of $100 \mathrm{mM}$ acetate (Riedel deHaen), pH 5.5 EDTA (Merck), 0.01\% Triton-X100 (Fluka) and 0.1\% glycerol (Carlo Erba). All solutions were manually spotted $(0.5 \mu \mathrm{l}$ per spot, spot diameter $=1 \mathrm{~mm}$ ) on PEO-like coated silicon wafers for SEM analysis and on glass slides cell attachment studies. The patterned samples were incubated for at least 15 minutes until the evaporation of the solvents. Samples were then thoroughly and carefully rinsed with ultrapure water and dried with nitrogen before further use.

\section{Cell attachment on Au NPs patterned samples}

A549 human lung epithelial cells (ATCC) were cultured under standard cell culture conditions $\left(37{ }^{\circ} \mathrm{C}, 5 \% \mathrm{CO}_{2}\right.$ and $95 \%$ humidity) in F12+GlutaMAX ${ }^{\text {TM}}-$ I medium (Life Technologies) complemented with $10 \%$ fetal calf serum (Life Technologies), $1 \%$ penicillin-streptomycin (Life Technologies) and 1\% HEPES buffer (Life Technologies). For routine culture the cells were grown in $75 \mathrm{~cm}^{2}$ flasks (Falcon) and passaged every 2-3 days when the cell layer was subconfluent.

For cell array generation, the fibronectin and Au NPspatterned glass slides were placed on the bottom of the wells in a 24-well culture plate (Falcon) and sterilized by exposure to ultraviolet radiation for 10 minutes prior to cell seeding. The A549 cells were washed with PBS, detached from the flasks using trypsin, centrifuged, re-suspended in culture medium and plated on the patterned samples at a cell density of $1 \times 10^{5}$ cells per well ( $500 \mu \mathrm{l}$ cell culture medium per well). The samples were incubated overnight in a cell culture incubator $\left(37^{\circ} \mathrm{C}, 5 \%\right.$ $\mathrm{CO}_{2}$ ). After overnight incubation, non-adherent cells were removed by gentle washing with warmed cell culture medium. Adherent cells were stained with propidium iodide $(5 \mu \mathrm{M}$, ex/em 535/617 nm) and Hoescht $33342(1 \mu \mathrm{M}$, ex/em 350/461 nm) for 5 minutes and carefully washed with PBS. The viability of A549 cells attached on the spots was assessed qualitatively under the Axiovert $200 \mathrm{M}$ microscope (Carl Zeiss; Jena, Germany) using $10 \times$ objective lens. Bright field and fluorescent images were obtained using the MosaiX module of the Axiovision 4.8 software (Carl Zeiss; Jena, Germany).

\section{Abbreviations}

$\begin{array}{ll}\text { Au NPs } & \text { Gold nanoparticles } \\ \text { PEO } & \text { Poly(ethyleneoxide) } \\ \text { RSA } & \text { Random sequential adsorption } \\ \text { DLVO } & \text { Derjaguin and Landau, Verwey and Overbeek } \\ \text { SEM } & \text { Scanning electron microscopy } \\ \text { SPR } & \text { Surface plasmon resonance } \\ \text { PI } & \text { Propidium iodide } \\ \text { QCM- } & \text { Quartz crystal microbalance with dissipation } \\ \text { D } & \text { monitoring } \\ \text { SAMs } & \text { Self-assembled monolayer } \\ \text { EDC } & \text { Ethyl(dimethylaminopropyl) carbodiimide } \\ \text { NHS } & N \text {-Hydroxysuccinimide } \\ \text { IgG } & \text { Immunoglobulines G }\end{array}$

\section{References}

1 N. L. Rosi and C. A. Mirkin, Nanostructures in biodiagnostics, Chem. Rev., 2005, 105, 1547-1562.

2 A. N. Shipway, E. Katz and I. Willner, Nanoparticle arrays on surfaces for electronic, optical, and sensor applications, ChemPhysChem, 2000, 1, 18-52.

3 B. D. Gates, Q. Xu, M. Stewart, D. Ryan, C. G. Willson and G. M. Whitesides, New Approaches to Nanofabrication: Molding, Printing, and Other Techniques, Chem. Rev., 2005, 105, 1171-1196.

4 P. K. Jain, W. Huang and M. A. El-Sayed, On the Universal Scaling Behavior of the Distance Decay of Plasmon Coupling in Metal Nanoparticle Pairs: A Plasmon Ruler Equation, Nano Lett., 2007, 7, 2080-2088.

5 A. M. Funston, C. Novo, T. J. Davis and P. Mulvaney, Plasmon Coupling of Gold Nanorods at Short Distances and in Different Geometries, Nano Lett., 2009, 9, 1651-1658.

6 I. Platzman, C. A. Muth, C. Lee-Thedieck, D. Pallarola, R. Atanasova, I. Louban, E. Altrock and J. P. Spatz, Surface properties of nanostructured bio-active interfaces: Impacts of surface stiffness and topography on cell-surface interactions, RSC Adv., 2013, 3(32), 13293-13303.

7 J. Groll, K. Albrecht, P. Gasteier, S. Riethmueller, U. Ziener and M. Moeller, Nanostructured Ordering of Fluorescent Markers and Single Proteins on Substrates, ChemBioChem, 2005, 6, 1782-1787.

8 D. Pallarola, A. Bochen, H. Boehm, F. Rechenmacher, T. R. Sobahi, J. P. Spatz and H. Kessler, Interface immobilization chemistry of cRGD-based peptides regulates integrin mediated cell adhesion, Adv. Funct. Mater., 2014, 24(7), 943-956.

9 E. A. Cavalcanti-Adam, A. Micoulet, J. Blümmel, J. Auernheimer, H. Kessler and J. P. Spatz, Lateral spacing of integrin ligands influences cell spreading and focal adhesion assembly, Eur. J. Cell Biol., 2006, 85, 219-224.

10 F. Brétagnol, M. Lejeune, A. Papadopoulou-Bouraoui, M. Hasiwa, H. Rauscher, G. Ceccone, P. Colpo and F. Rossi, Fouling and non-fouling surfaces produced by plasma polymerization of ethylene oxide monomer, Acta Biomater., 2006, 2, 165-172.

11 A. Ruiz, L. Ceriotti, L. Buzanska, M. Hasiwa, F. Brétagnol, G. Ceccone, D. Gilliland, H. Rauschera, S. Coecke, P. Colpo and F. Rossi, Controlled micropatterning of biomolecules for cell culturing, Microelectron. Eng., 2007, 84, 1733-1736.

12 A. Ruiz, M. Zychowicz, L. Ceriotti, D. Mehn, L. Sirghi, H. Rauscher, I. Mannelli, P. Colpo, L. Buzanska and F. Rossi, Microcontact printing and microspotting as methods for direct protein patterning on plasma deposited polyethylene oxide: application to stem cell patterning, Biomed. Microdevices, 2013, 15, 495-507.

$13 \mathrm{~J}$. W. Evans, Random and cooperative sequential adsorption, Rev. Mod. Phys., 1993, 65, 1281.

$14 \mathrm{~J}$. Israelachvili and R. Pashley, The hydrophobic interaction is long range, decaying exponentially with distance, Nature, 1982, 300, 341-342. 
15 L. Xu, G. Han, J. Hu, Y. He, J. Pan, Y. Li and J. Xianga, Hydrophobic coating- and surface active solvent-mediated self-assembly of charged gold and silver nanoparticles at water-air and water-oil interfaces, Phys. Chem. Chem. Phys., 2009, 11, 6490-6497.

16 E. Thormann, A. C. Simonsen, P. L. Hansen and O. G. Mouritsen, Interactions between a Polystyrene Particle and Hydrophilic and Hydrophobic Surfaces in Aqueous Solutions, Langmuir, 2008, 24, 7278-7284.
17 M. J. Pérez-Roldan, A. Parracino, G. Ceccone, P. Colpo and F. Rossi, Interactions of serum derived proteins with submicrometer structured surfaces, Plasma Processes Polym., 2014, 11(6), 577-587.

18 F. L. Yap and Y. Zhang, Protein and cell micropatterning and its integration with micro/nanoparticles assembly, Biosens. Bioelectron., 2007, 22, 775-788.

19 J. El-Ali, P. K. Sorger and K. F. Jensen, Cells on chips, Nature, 2006, 442, 403-411. 